\title{
Initial Communication Survey Results for the Hanford Environmental Dose Reconstruction Project
}

D. M. Beck

\section{March 1991}
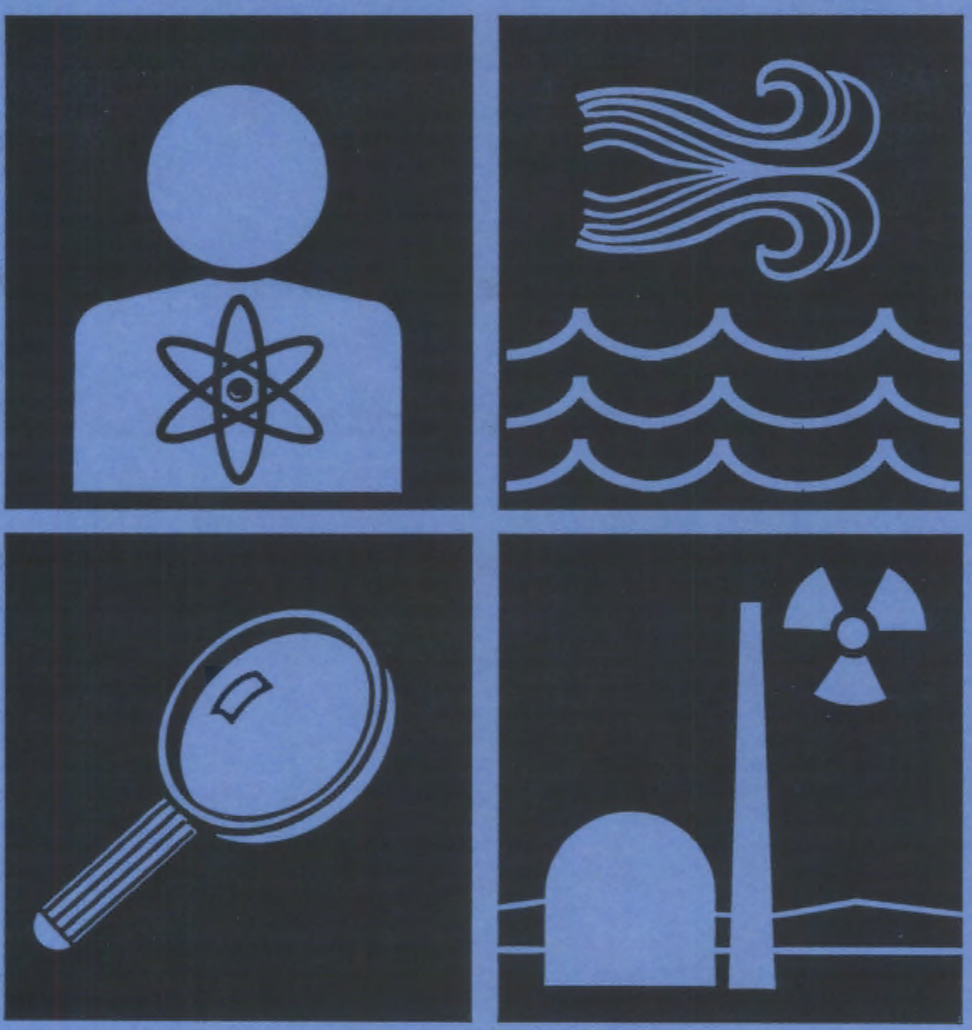

Prepared for the Technical Steering Panel 


\section{DISCLAIMER}

This report was prepared under the direction of the HANFORD ENVIRONMENTAL DOSE RECONSTRUCTION PROJECT Technical Steering Panel by Battelle Memorial Institute's Pacific Northwest Laboratories operating the Pacific Northwest Laboratory for the U.S. Department of Energy (DOE). While funding for the work was provided by DOE, the work is not under DOE direction or control. The views and opinions of the authors expressed in this document do not necessarily reflect those of the United States Government or any agency thereof. Reference herein to any specific commercial product, process or service by trade name, trademark, manufacturer or otherwise does not necessarily constitute or imply its endorsement, recommendation or favoring by the U.S. Government or any agency thereof, nor by Battelle Memorial Institute.

Printed in the United States of America

Available to DOE and DOE contractors from the

Office of Scientific and Technical Information, P.O. Box 62, Oak Ridge, TN 37831 ; prices available from (615) 576-8401. FTS 626-8401.

Available to the public from the National Technical Information Service, U.S. Department of Commerce, 5285 Port Royal Rd., Springfield, VA 22161. 
INITIAL COMMUNICATION SURVEY RESULTS FOR THE HANFORD ENVIRONMENTAL DOSE RECONSTRUCTION PROJECT

D. M. Beck

March 1991

Prepared for the Technical Steering Pane1

Pacific Northwest Laboratory Richland, Washington 99352 
INITIAL COMMUNICATION SURVEY RESULTS FOR THE HANFORD ENVIRONMENTAL DOSE RECONSTRUCTION PROJECT

March 1991

This document has been reviewed and approved by the Technical Steering Panel.

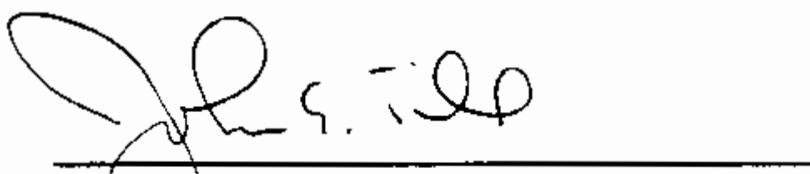

Johln E. Till, Chairman

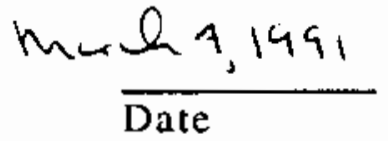

Technical Steering Panel

Hanford Environmental

Dose Reconstruction Project 



\section{SUMMARY}

To support the public communication efforts of the Technical Steering Panel of the Hanford Environmenta] Dose Reconstruction (HEDR) Project, a public survey was conducted. The survey was intended to provide information about the public's knowledge and interest in the project and the best ways to communicate project results.

Questions about the project were included as part of an omnibus survey conducted by Washington State University. The survey was conducted by phone to Washington State residents in the spring of 1990. This report gives the HEDR-related questions and summary data of responses.

Questions associated with the HEDR Project were grouped into four categories:

- knowledge of the HEDR Project

- interest in the project

- preferred ways of receiving information about the project (including public information meetings, a newsletter mailed to homes, presentations to civic groups in the respondent's community, a computer bulletin board respondent could access with a modem, information displays at public buildings and shopping malls, and an information video sent to respondent)

- level of concern over past exposure from Hanford operations.

Questions about whom state residents are most likely to trust about radiation issues were also part of the omnibus survey, and responses are included in this report. 



\section{CONTENTS}

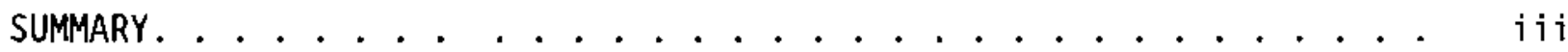

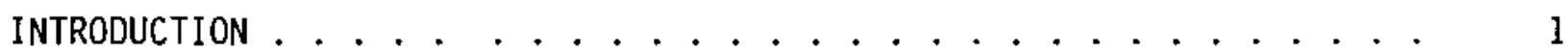

SURVEY DESIGN AND IMPLEMENTATION . . . . . . . . . . . . . . . . . 3

SURVEY RESULTS . . . . . . . . . . . . . . . . . . . . 5

KNOWLEDGE OF THE HEDR PROJECT . . . . . . . . . . . . . . . 5

INTEREST IN THE HEDR PROJECT . . . . . . . . . . . . . . 6

CONCERN OVER PAST EXPOSURE .................... 9

WHOM RESPONDENTS TRUST . . . . . . . . . . . . . . . 10

DEMOGRAPHICS. . . . . . . . . . . . . . . . . . 12 


\section{INTRODUCTION}

The Hanford Environmental Dose Reconstruction (HEDR) Project Technical Steering Panel (TSP) is charged with the responsibility to communicate project results to the general public. To support the TSP's communication efforts, a survey of Washington State residents was conducted to solicit citizen knowledge and to determine interest in the HEDR Project and to determine the best ways to communicate results to the public. The purpose of this document is to present the initial results of this survey. Survey design and implementation is discussed in Section 2.0, while results of the survey are presented in Section 3.0. Sections detailing an analysis of the survey data and survey conclusions will be added at a later date. 

The survey was conducted as part of an omnibus survey conducted by the Social and Economic Sciences Research Center (SESRC) at Washington State University (WSU). The HEDR questions were developed by PNL staff using the Total Design Method developed by Don Dillman at WSU. The survey was conducted by telephone to residents of Washington State between March 27 and Apri] 30, 1990. The sample was drawn to obtain a representative sample of the entire population of Washington State using a random digit dialing (RDD) sampling frame. Pretests of the questionnaire were conducted on March 21, 1990 to resolve any problems with wording. The data collected in the pre-tests are not included in the final results. The interviews were conducted from the Public Opinion Laboratory of the SESRC using a microcomputer-assisted telephone interviewing (MATI) facility. The cooperation rate (the ratio of completed interviews to the total number of completed interviews, plus refusals, plus partially completed interviews) was $56.6 \%$. 


\section{SURVEY RESULTS}

KNOWLEDGE OF. THE HEDR PROJECT

Questions 1-5 were asked to determine what percentage of respondents had heard of the HEDR Project. One of the projects asked about, the Atmospheric Reclamation and Reconstruction Project, is a fictitious project. It was asked to get a crude indicator of what percentage of respondents would say they heard about a particular project even though in fact they had not. This type of bias is referred to as "social desirability bias."

Q1-Q5. At the Hanford Site a variety of research projects are under way. We would Tike to know if people such as yourself have heard about any of these projects. I'm going to read you a list of projects and I'd like you to tell me if you have SEEN, HEARD, or READ anything about the project. The first project is the $\mathrm{N}$-Reactor shutdown. Have you seen, heard, or read anything about this project?

\section{Q1. N-REACTOR SHUTDOWN PROJECT}

\begin{tabular}{lccccc} 
VAR71 & Frequency & Percent & $\begin{array}{c}\text { Cumulative } \\
\text { Frequency }\end{array}$ & $\begin{array}{c}\text { Cumulative } \\
\text { Percent }\end{array}$ \\
\cline { 1 - 2 } Yes & 367 & 70.3 & & 367 & 70.3 \\
No & 155 & 29.7 & & 522 & 100.0
\end{tabular}

Frequency Missing $=25$

Q2. MOLECULAR SCIENCES RESEARCH PROJECT

$\begin{array}{lccccc}\text { VAR72 } & \text { Frequency } & \text { Percent } & \begin{array}{c}\text { Cumulative } \\ \text { Frequency }\end{array} & \begin{array}{c}\text { Cumulative } \\ \text { Percent }\end{array} \\ \text { Yes } & 84 & 16.2 & & 84 & \\ \text { No } & 434 & 83.8 & & 518 & 16.2 \\ \text { Frequency Missing } & =29 & & & \end{array}$




\section{Q3. DOSE RECONSTRUCTION PROJECT}

\begin{tabular}{|c|c|c|c|c|}
\hline VAR73 & Frequency & Percent & $\begin{array}{l}\text { Cumulative } \\
\text { Frequency }\end{array}$ & $\begin{array}{l}\text { Cumulative } \\
\text { Percent }\end{array}$ \\
\hline $\begin{array}{l}\text { Yes } \\
\text { No }\end{array}$ & $\begin{array}{l}145 \\
371\end{array}$ & $\begin{array}{l}28.1 \\
71.9\end{array}$ & $\begin{array}{l}145 \\
516\end{array}$ & $\begin{array}{r}28.1 \\
100.0\end{array}$ \\
\hline
\end{tabular}

\section{Q4. FAST FLUX TEST FACILITY PROJECT}

$\begin{array}{cccccc}\begin{array}{cccc}\text { VAR74 } \\ \text { Fes }\end{array} & \text { Frequency } & \text { Percent } & \begin{array}{c}\text { Cumulative } \\ \text { Frequency }\end{array} & \begin{array}{c}\text { Cumulative } \\ \text { Percent }\end{array} \\ \text { No } & 125 & 24.1 & & 125 & \\ \text { No } & 393 & 75.9 & & 518 & 100.0\end{array}$

Frequency Missing $=29$

Q5. ATMOSPHERIC RECLAMATION/RECONSTRUCTION

\begin{tabular}{|c|c|c|c|c|}
\hline VAR75 & Frequency & Percent & $\begin{array}{l}\text { Cumulative } \\
\text { Frequency }\end{array}$ & $\begin{array}{l}\text { Cumulative } \\
\text { Percent }\end{array}$ \\
\hline $\begin{array}{l}\text { Yes } \\
\text { No }\end{array}$ & $\begin{array}{r}77 \\
441\end{array}$ & $\begin{array}{l}14.9 \\
85.1\end{array}$ & $\begin{array}{r}77 \\
518\end{array}$ & $\begin{array}{r}14.9 \\
100.0\end{array}$ \\
\hline
\end{tabular}

\section{INTEREST IN THE HEDR PROJECT}

Now I would like to ask you a few questions about an ongoing project at Hanford called the Environmental Dose Reconstruction Project. The purpose of this project is to determine what level of radiation people in the state may have been exposed to from 1944-1970 due to Hanford activities.

Q-6. Which of the following best describes your interest in receiving more information about the Hanford Environmental Dose Reconstruction Project? 


\begin{tabular}{lccccc}
\multicolumn{1}{c}{ VAR76 } & Frequency & Percent & $\begin{array}{c}\text { Cumulative } \\
\text { Frequency }\end{array}$ & $\begin{array}{c}\text { Cumulative } \\
\text { Percent }\end{array}$ \\
\cline { 1 - 1 } \cline { 5 - 6 } VERY INTERESTED & & 16 & 61.9 & 166 & 31.9 \\
SOMEWHAT INT & 268 & & 51.5 & 434 & 83.5 \\
NOT INT & 86 & 16.5 & 520 & 100.0 \\
Frequency Missing & $=27$ & & & &
\end{tabular}

Q7-Q14. There are a variety of ways of getting information about the Hanford Environmental Dose Reconstruction Project to interested members of the public, such as yourself. For each of the following ways, tell me whether it would be very useful, somewhat useful, of little use, or of no use, to you personally. The first way is THROUGH PUBLIC INFORMATION MEETINGS HELD IN THE TRI-CITIES AREA. Would you personally find this way of obtaining information very useful, somewhat useful, little use, or of no use at all?

\section{Q7. PUBLIC INFO MEETINGS IN TRI-CITIES}

\begin{tabular}{|c|c|c|c|c|}
\hline VAR77 & Frequency & Percent & $\begin{array}{l}\text { Cumulat ive } \\
\text { Frequency }\end{array}$ & $\begin{array}{l}\text { Cumulative } \\
\text { Percent }\end{array}$ \\
\hline $\begin{array}{l}\text { VERY USEFUL } \\
\text { SOMEWHAT USEFUL } \\
\text { LITTLE USE } \\
\text { NO USE }\end{array}$ & $\begin{array}{r}41 \\
55 \\
114 \\
223\end{array}$ & $\begin{array}{r}9.5 \\
12.7 \\
26.3 \\
51.5\end{array}$ & $\begin{array}{r}41 \\
96 \\
210 \\
433\end{array}$ & $\begin{array}{r}9.5 \\
22.2 \\
48.5 \\
100.0\end{array}$ \\
\hline
\end{tabular}

Frequency Missing $=114$

Q8. PUBLIC INFO MEETINGS IN YOUR TOWN

\begin{tabular}{|c|c|c|c|c|}
\hline VAR78 & Frequency & Percent & $\begin{array}{l}\text { Cumulative } \\
\text { Erequency }\end{array}$ & $\begin{array}{l}\text { Cumulative } \\
\text { Percent }\end{array}$ \\
\hline $\begin{array}{l}\text { VERY USEFUL } \\
\text { SOMEWHAT USEFUL } \\
\text { LITTLE USE } \\
\text { NO USE }\end{array}$ & $\begin{array}{r}130 \\
187 \\
75 \\
42\end{array}$ & $\begin{array}{r}30.0 \\
43.1 \\
17.3 \\
9.7\end{array}$ & $\begin{array}{l}130 \\
317 \\
392 \\
434\end{array}$ & $\begin{array}{r}30.0 \\
73.0 \\
90.3 \\
100.0\end{array}$ \\
\hline
\end{tabular}


Q9. AN INFORMATION NEWSLETTER MAILED DIRECTLY TO YOUR HOME

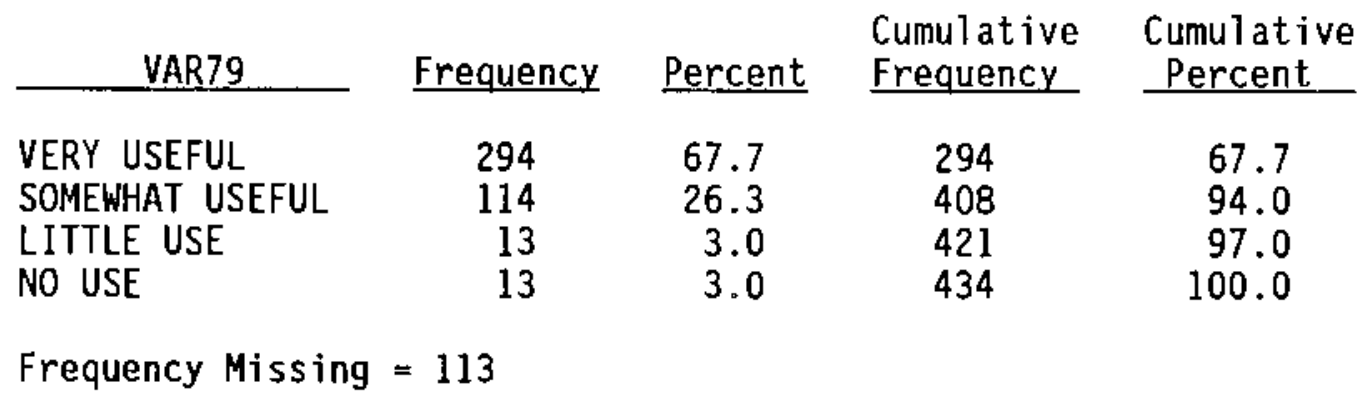

Q10. PRESENTATIONS TO CIVIC GROUPS IN YOUR COMMUNITY (e.g., KIWANIS, ROTARY, PTA, GRANGE, etc.)

\begin{tabular}{|c|c|c|c|c|}
\hline VARBO & Frequency & Percent & $\begin{array}{l}\text { Cumulative } \\
\text { Frequency }\end{array}$ & $\begin{array}{l}\text { Cumulative } \\
\text { Percent }\end{array}$ \\
\hline $\begin{array}{l}\text { VERY USEFUL } \\
\text { SOMEWHAT USEFUL } \\
\text { LITTLE USE } \\
\text { NO USE }\end{array}$ & $\begin{array}{r}103 \\
175 \\
86 \\
68\end{array}$ & $\begin{array}{l}23.8 \\
40.5 \\
19.9 \\
15.7\end{array}$ & $\begin{array}{l}103 \\
278 \\
364 \\
432\end{array}$ & $\begin{array}{r}23.8 \\
64.4 \\
84.3 \\
100.0\end{array}$ \\
\hline
\end{tabular}

Frequency Missing $=115$

Q11. A COMPUTER BULLETIN BOARD YOU COULD ACCESS WITH A MODEM

\begin{tabular}{lccccc}
\multicolumn{1}{c}{ VAR81 } & Frequency & Percent & $\begin{array}{c}\text { Cumulative } \\
\text { Frequency }\end{array}$ & $\begin{array}{c}\text { Cumulative } \\
\text { Percent }\end{array}$ \\
\cline { 1 - 1 } \cline { 5 - 6 } VERY USEFUL & & & & \\
SOMEWHAT USEFUL & 79 & 11.5 & & 49 & 11.5 \\
LITTLE USE & 88 & & 17.8 & 125 & 29.5 \\
NO USE & 214 & 50.1 & & 427 & 100.0 \\
Frequency Missing & $=120$ & & & &
\end{tabular}


Q12. INFORMATION DISPLAYS AT PUBLIC LIBRARIES AND OTHER PUBLIC BUILDINGS

\begin{tabular}{|c|c|c|c|c|}
\hline VAR82 & Frequency & Percent & $\begin{array}{l}\text { Cumulative } \\
\text { Frequency }\end{array}$ & $\begin{array}{l}\text { Cumulative } \\
\text { Percent }\end{array}$ \\
\hline $\begin{array}{l}\text { VERY USEFUL } \\
\text { SOMEWHAT USEFUL } \\
\text { LITTLE USE } \\
\text { NO USE }\end{array}$ & $\begin{array}{r}145 \\
171 \\
67 \\
49\end{array}$ & $\begin{array}{l}33.6 \\
39.6 \\
15.5 \\
11.3\end{array}$ & $\begin{array}{l}145 \\
316 \\
383 \\
432\end{array}$ & $\begin{array}{r}33.6 \\
73.1 \\
88.7 \\
100.0\end{array}$ \\
\hline
\end{tabular}

Frequency Missing $=115$

Q13. INFO DISPLAYS AT LOCAL SHOPPING MALLS

\begin{tabular}{|c|c|c|c|c|}
\hline VAR83 & Frequency & Percent & $\begin{array}{l}\text { Cumulative } \\
\text { Frequency }\end{array}$ & $\begin{array}{l}\text { Cumulative } \\
\text { Percent }\end{array}$ \\
\hline $\begin{array}{l}\text { VERY USEFUL } \\
\text { SOMEWHAT USEFUL } \\
\text { LITTLE USE } \\
\text { NO USE }\end{array}$ & $\begin{array}{r}112 \\
169 \\
79 \\
73\end{array}$ & $\begin{array}{l}25.9 \\
39.0 \\
18.2 \\
16.9\end{array}$ & $\begin{array}{l}112 \\
281 \\
360 \\
433\end{array}$ & $\begin{array}{r}25.9 \\
64.9 \\
83.1 \\
100.0\end{array}$ \\
\hline
\end{tabular}

Q14. AN INFORMATION VIDEO THAT COULD BE SENT TO YOU

\begin{tabular}{|c|c|c|c|c|}
\hline VAR84 & Frequency & Percent & $\begin{array}{l}\text { Cumulat ive } \\
\text { Frequency }\end{array}$ & $\begin{array}{l}\text { Cumulative } \\
\text { Percent }\end{array}$ \\
\hline $\begin{array}{l}\text { VERY USEFUL } \\
\text { SOMEWHAT USEFUL } \\
\text { LITYLE USE } \\
\text { NO USE }\end{array}$ & $\begin{array}{r}170 \\
130 \\
52 \\
79\end{array}$ & $\begin{array}{l}39.4 \\
30.2 \\
12.1 \\
18.3\end{array}$ & $\begin{array}{l}170 \\
300 \\
352 \\
431\end{array}$ & $\begin{array}{r}39.4 \\
69.6 \\
81.7 \\
100.0\end{array}$ \\
\hline
\end{tabular}

Frequency Missing $=116$

\section{CONCERN OVER PAST EXPOSURE}

Q-15. Due to operations at Hanford from 1944 to 1970 , radiation was released to the environment. Some people are concerned that they may have been exposed to this radiation, while others are not concerned. Which of the following best describes YOUR concern over past exposure? 


\begin{tabular}{|c|c|c|c|c|}
\hline VAR86 & Frequency & Percent & $\begin{array}{l}\text { Cumulative } \\
\text { Frequency }\end{array}$ & $\begin{array}{l}\text { Cumulat ive } \\
\text { Percent }\end{array}$ \\
\hline $\begin{array}{l}\text { VERY CONCERNED } \\
\text { SOMEWHAT CONCERN } \\
\text { NOT CONCERNED } \\
\text { NOT LIVE IN WASH }\end{array}$ & $\begin{array}{r}95 \\
161 \\
219 \\
38\end{array}$ & $\begin{array}{r}18.5 \\
31.4 \\
42.7 \\
7.4\end{array}$ & $\begin{array}{r}95 \\
256 \\
475 \\
513\end{array}$ & $\begin{array}{r}18.5 \\
49.9 \\
92.6 \\
100.0\end{array}$ \\
\hline
\end{tabular}

\section{WHOM RESPONDENTS TRUST}

The following questions were asked as part of a series of questions on radioactive waste and waste disposal. However, because the answers suggest whom state residents are most likely to trust about radiation issues, they are reproduced here.

Q16-Q23. When it comes to providing information about radioactive waste and waste disposal, how much would you trust each of the following to provide reliable information? For each one, tell me if you would trust them VERY MUCH, A LITTLE, NOT TOO MUCH, OR NOT AT ALL. The first one is FEDERAL NUCLEAR AGENCIES. Would you trust them very much, a little, not too much, or not at al1?

Q16. FEDERAL NUCLEAR AGENCIES

\begin{tabular}{|c|c|c|c|c|}
\hline VAR62 & Frequency & Percent & $\begin{array}{l}\text { Cumulative } \\
\text { Frequency }\end{array}$ & $\begin{array}{l}\text { Cumulative } \\
\text { Percent } \\
\end{array}$ \\
\hline $\begin{array}{l}\text { VERY MUCH } \\
\text { A LITTLE } \\
\text { NOT TOO MUCH } \\
\text { NOT AT ALL }\end{array}$ & $\begin{array}{r}76 \\
229 \\
148 \\
61\end{array}$ & $\begin{array}{l}14.8 \\
44.6 \\
28.8 \\
11.9\end{array}$ & $\begin{array}{r}76 \\
305 \\
453 \\
514\end{array}$ & $\begin{array}{r}14.8 \\
59.3 \\
88.1 \\
100.0\end{array}$ \\
\hline
\end{tabular}


Q17. UNIVERSITY SCIENTISTS

\begin{tabular}{|c|c|c|c|c|}
\hline VAR69 & Frequency & Percent & $\begin{array}{l}\text { Cumulative } \\
\text { Frequency }\end{array}$ & $\begin{array}{l}\text { Cumul at ive } \\
\text { Percent }\end{array}$ \\
\hline $\begin{array}{l}\text { VERY MUCH } \\
\text { A LITTLE } \\
\text { NOT TOO MUCH } \\
\text { NOT AT ALL }\end{array}$ & $\begin{array}{r}337 \\
152 \\
11 \\
13\end{array}$ & $\begin{array}{r}65.7 \\
29.6 \\
2.1 \\
2.5\end{array}$ & $\begin{array}{l}337 \\
489 \\
500 \\
513\end{array}$ & $\begin{array}{r}65.7 \\
95.3 \\
97.5 \\
100.0\end{array}$ \\
\hline
\end{tabular}

Q18. INDEPENDENT CITIZEN GROUPS

\begin{tabular}{|c|c|c|c|c|}
\hline VAR63 & Frequency & Percent & $\begin{array}{l}\text { Cumulative } \\
\text { Frequency }\end{array}$ & $\begin{array}{l}\text { Cumulative } \\
\text { Percent }\end{array}$ \\
\hline $\begin{array}{l}\text { VERY MUCH } \\
\text { A LITTLE } \\
\text { NOT TOO MUCH } \\
\text { NOT AT ALL }\end{array}$ & $\begin{array}{r}181 \\
230 \\
70 \\
35\end{array}$ & $\begin{array}{r}35.1 \\
44.6 \\
13.6 \\
6.8\end{array}$ & $\begin{array}{l}181 \\
411 \\
481 \\
516\end{array}$ & $\begin{array}{r}35.1 \\
79.7 \\
93.2 \\
100.0\end{array}$ \\
\hline
\end{tabular}

Q19. FEDERAL ENVIRONMENTAL AGENCIES

\begin{tabular}{|c|c|c|c|c|}
\hline VAR64 & Frequency & Percent & $\begin{array}{l}\text { Cumulative } \\
\text { Frequency }\end{array}$ & $\begin{array}{l}\text { Cumulative } \\
\text { Percent }\end{array}$ \\
\hline $\begin{array}{l}\text { VERY MUCH } \\
\text { A LITTLE } \\
\text { NOT TOO MUCH } \\
\text { NOT AT ALL }\end{array}$ & $\begin{array}{r}135 \\
245 \\
99 \\
37\end{array}$ & $\begin{array}{r}26.2 \\
47.5 \\
19.2 \\
7.2\end{array}$ & $\begin{array}{l}135 \\
380 \\
479 \\
516\end{array}$ & $\begin{array}{r}26.2 \\
73.6 \\
92.8 \\
100.0\end{array}$ \\
\hline
\end{tabular}

Q20. UTILITY OFFICIALS IN YOUR COMMUNITY

\begin{tabular}{|c|c|c|c|c|}
\hline VAR65 & Frequency & Percent & $\begin{array}{l}\text { Cumulative } \\
\text { Frequency }\end{array}$ & $\begin{array}{l}\text { Cumul at ive } \\
\text { Percent }\end{array}$ \\
\hline $\begin{array}{l}\text { VERY MUCH } \\
\text { A LITTLE } \\
\text { NOT TOO MUCH } \\
\text { NOT AT ALL }\end{array}$ & $\begin{array}{r}102 \\
234 \\
107 \\
69\end{array}$ & $\begin{array}{l}19.9 \\
45.7 \\
20.9 \\
13.5\end{array}$ & $\begin{array}{l}102 \\
336 \\
443 \\
512\end{array}$ & $\begin{array}{r}19.9 \\
65.6 \\
86.5 \\
100.0\end{array}$ \\
\hline
\end{tabular}


Q21. WASHINGTON STATE AGENCIES

\begin{tabular}{|c|c|c|c|c|}
\hline VAR66 & Frequency & Percent & $\begin{array}{l}\text { Cumul at ive } \\
\text { Frequency }\end{array}$ & $\begin{array}{l}\text { Cumul at ive } \\
\text { Percent }\end{array}$ \\
\hline $\begin{array}{l}\text { VERY MUCH } \\
\text { A LITTLE } \\
\text { NOT TOO MUCH } \\
\text { NOT AT ALL }\end{array}$ & $\begin{array}{r}148 \\
266 \\
65 \\
29\end{array}$ & $\begin{array}{r}29.1 \\
52.4 \\
12.8 \\
5.7\end{array}$ & $\begin{array}{l}148 \\
414 \\
479 \\
508\end{array}$ & $\begin{array}{r}29.1 \\
81.5 \\
94.3 \\
100.0\end{array}$ \\
\hline
\end{tabular}

Q22. OFFICIALS IN YOUR COUNTY AND CITY

\begin{tabular}{|c|c|c|c|c|}
\hline VAR67 & Frequency & Percent & $\begin{array}{l}\text { Cumulative } \\
\text { Frequency }\end{array}$ & $\begin{array}{l}\text { Cumulative } \\
\text { Percent }\end{array}$ \\
\hline $\begin{array}{l}\text { VERY MUCH } \\
\text { A LITTLE } \\
\text { NOT TOO MUCH } \\
\text { NOT AT ALL }\end{array}$ & $\begin{array}{r}114 \\
262 \\
86 \\
52\end{array}$ & $\begin{array}{l}22.2 \\
51.0 \\
16.7 \\
10.1\end{array}$ & $\begin{array}{l}114 \\
376 \\
462 \\
514\end{array}$ & $\begin{array}{r}22.2 \\
73.2 \\
89.9 \\
100.0\end{array}$ \\
\hline
\end{tabular}

Q23. ENVIRONMENTAL ORGANIZATIONS

\begin{tabular}{|c|c|c|c|c|}
\hline VAR68. & Frequency & Percent & $\begin{array}{l}\text { Cumulat ive } \\
\text { Frequency }\end{array}$ & $\begin{array}{l}\text { Cumulative } \\
\text { Percent }\end{array}$ \\
\hline $\begin{array}{l}\text { VERY MUCH } \\
\text { A LITTLE } \\
\text { NOT TOO MUCH } \\
\text { NOT AT ALL. }\end{array}$ & $\begin{array}{r}210 \\
223 \\
53 \\
25\end{array}$ & $\begin{array}{r}41.1 \\
43.6 \\
10.4 \\
4.9\end{array}$ & $\begin{array}{l}210 \\
433 \\
486 \\
511\end{array}$ & $\begin{array}{r}41.1 \\
84.7 \\
95.1 \\
100.0\end{array}$ \\
\hline
\end{tabular}

DEMOGRAPHICS

Q24. SEX

\begin{tabular}{lcccc}
\multicolumn{1}{c}{ VAR87 } & Erequency & Percent & $\begin{array}{c}\text { Cumulative } \\
\text { Frequency }\end{array}$ & $\begin{array}{c}\text { Cumulative } \\
\text { Percent }\end{array}$ \\
\cline { 1 - 1 } & & & & \\
MALE & 245 & 47.4 & 245 & 47.4 \\
FEMALE & 272 & 52.6 & 517 & 100.0 \\
Frequency Missing & $=30$ & & &
\end{tabular}


Q25. EDUCATION

\begin{tabular}{|c|c|c|c|c|}
\hline VAR113 & Frequency & Percent & $\begin{array}{l}\text { Cumulative } \\
\text { Frequency }\end{array}$ & $\begin{array}{l}\text { Cumulative } \\
\text { Percent }\end{array}$ \\
\hline $\begin{array}{l}\text { MISSING } \\
\text { SKIP }\end{array}$ & $\begin{array}{r}3 \\
29\end{array}$ & & & \\
\hline $\begin{array}{l}\text { NO HIGH SCHOOL } \\
\text { DIPLOMA }\end{array}$ & 57 & 11.1 & 57 & 11.1 \\
\hline $\begin{array}{l}\text { HS GRAD/GED } \\
\text { SOME COLLEGE } \\
\text { ASSOC DEGREE } \\
\text { BA, AB OR BS } \\
\text { PROF. MA OR PHD }\end{array}$ & $\begin{array}{r}115 \\
132 \\
43 \\
112 \\
56\end{array}$ & $\begin{array}{r}22.3 \\
25.6 \\
8.3 \\
21.7 \\
10.9\end{array}$ & $\begin{array}{l}172 \\
304 \\
347 \\
459 \\
515\end{array}$ & $\begin{array}{r}33.4 \\
59.0 \\
67.4 \\
89.1 \\
100.0\end{array}$ \\
\hline
\end{tabular}

Q26. RACE

\begin{tabular}{|c|c|c|c|c|}
\hline VARI14 & Frequency & Percent & $\begin{array}{l}\text { Cumulat ive } \\
\text { Frequency }\end{array}$ & $\begin{array}{l}\text { Cumulative } \\
\text { Percent }\end{array}$ \\
\hline $\begin{array}{l}\text { WHITE } \\
\text { BLACK } \\
\text { AM IND } \\
\text { ASIAN / ISL } \\
\text { OTHER }\end{array}$ & $\begin{array}{r}484 \\
4 \\
6 \\
6 \\
17\end{array}$ & $\begin{array}{r}93.6 \\
0.8 \\
1.2 \\
1.2 \\
3.3\end{array}$ & $\begin{array}{l}484 \\
488 \\
494 \\
500 \\
517\end{array}$ & $\begin{array}{r}93.6 \\
94.4 \\
95.6 \\
96.7 \\
100.0\end{array}$ \\
\hline
\end{tabular}

Q27. AGE

\begin{tabular}{|c|c|c|c|c|}
\hline VAR87 & Frequency & Percent & $\begin{array}{l}\text { Cumulative } \\
\text { Erequency }\end{array}$ & $\begin{array}{l}\text { Cumulat ive } \\
\text { Percent }\end{array}$ \\
\hline $\begin{array}{l}\text { MISSING } \\
18 \text { T0 } 29 \\
30 \text { T0 } 39 \\
40 \text { to } 49 \\
50 \text { to } 59 \\
60 \text { to } 69 \\
70 \text { AND OVER }\end{array}$ & $\begin{array}{r}41 \\
89 \\
138 \\
101 \\
60 \\
63 \\
55\end{array}$ & $\begin{array}{l}17.6 \\
27.3 \\
20.0 \\
11.9 \\
12.5 \\
10.9\end{array}$ & $\begin{array}{r}89 \\
227 \\
328 \\
388 \\
451 \\
506\end{array}$ & $\begin{array}{r}17.6 \\
44.9 \\
64.8 \\
76.7 \\
89.1 \\
100.0\end{array}$ \\
\hline
\end{tabular}


Q28. INCOME

\begin{tabular}{lcccc}
\multicolumn{1}{c}{ VAR87 } & Frequency & Percent & $\begin{array}{c}\text { Cumulative } \\
\text { Frequency }\end{array}$ & $\begin{array}{c}\text { Cumulative } \\
\text { Percent }\end{array}$ \\
\cline { 1 - 1 } $\begin{array}{l}\text { DON'T KNOW } \\
\text { MISSING }\end{array}$ & 4 & & & \\
REFUSE & 15 & & & \\
SKIP & 17 & & & \\
LESS THAN $\$ 10000$ & 29 & & & \\
$\$ 10000$ TO $\$ 20000$ & 98 & 20.4 & 31 & 6.4 \\
$\$ 20000$ T0 $\$ 30000$ & 100 & 20.7 & 129 & 26.8 \\
$\$ 30000$ T0 $\$ 40000$ & 89 & 18.5 & 318 & 67.5 \\
$\$ 40000$ T0 $\$ 50000$ & 53 & 11.0 & 371 & 77.0 \\
$\$ 50000$ T0 $\$ 60000$ & 45 & 9.3 & 416 & 86.3 \\
$\$ 60000$ TO $\$ 80000$ & 31 & 6.4 & 447 & 92.7 \\
OVER $\$ 80000$ & 35 & 7.3 & 482 & 100.0
\end{tabular}




\section{DISTRIBUTION}

No. of

Copies

OFFSITE

\section{Technical Steering Pane1}

D. S. Barth

University of Nevada

4505 Maryland Parkway

Las Vegas, NV 89154

W. A. Bishop

2503 Wedgewood Court SE

Olympia, WA 98501

M. L. Blazek

Oregon Department of Energy

625 Marion Street N.E.

Salem, OR 97310

G. G. Caldwell

Director

Tulsa City-County Health Dept

4616 East 15th Street

Tulsa, OK 74112

S. N. Davis

Dept. of Hydrology \& Water

Resources

Bldg. 11

University of Arizona

Tucson, AZ 85721

N. J. Germond

224 Iron Mountain B7vd.

Lake Ozwego, OR 97034

P. C. Klingeman

Civil Engineering Dept.

Oregon State University

Corvallis, OR 97331-2302
No. of

Copies

\section{K. J. Kopecky}

Fred Hutchinson Cancer Research Center

1124 Columbia Street

Seattle, WA 98104

R. L. Morrill

Dept. of Geography

DP-10

University of Washington

Seattle, WA 98195

A. H. Murphy

$\mathrm{C} / \mathrm{O} \mathrm{Cl}$ imate Analyses Center

National Weather Service, NOAA

W/NMC51, WWB, Room 604

Washington, DC 20233

$\checkmark$. Nguyen

EWA, Ine .

133 lst Ave. N.

Minneapolis, MN 55401

D. W. Price

Agricultural Economics

Hulbert Hall Room 211

Washington State University

Pullman, WA 99164-6210

M. A. Robkin

Radiological Sciences SB-75

University of Washington

Seattle, WA 98195

G. S. Roessler

Rt. 1, Box 139H

Elysian, MN 56028 
No. of

Copies

B. Shleien 2421 Homestead Drive Silver Springs, MD 20902

A. P. Slickpoo, Sr. P. 0. Box 331 $809 \mathrm{Nez}$ Perce Lane Kamiah, ID 83536

J. E. Till

Rt. 2 Box 122

Neeses, SC 29107

D. E. Walker, Jr.

P.0. Box 4147

Boulder, C0 80306

2 DOE Office of Scientific and Technical Information Technical Information Center P.0. Box 62

Oak Ridge, TN 37830

10 J. S. Stohr

Office of Nuclear Waste Mgmt. Department of Ecology 99 South Sound Center Mail Stop PV-11

01 ympia, WA 98504
No. of

Copies

ONSITE

\section{DOE Richland Operations}

R. F. Brich, SED A5-55

26 Pacific Northwest Laboratory

D. M. Beck (5)

K6-57

W. A. Glass

$\mathrm{K} 1-40$

S. P. Gydesen

P8-55

G. L. Harvey

$\mathrm{K} 1-55$

C. W. Holmes

$K 6-66$

A. H. McMakin

$\mathrm{K} 6-86$

R. E. Rhoads

$\mathrm{K} 6-64$

W. L. Templeton

$\mathrm{K} 1-30$

Project Office Files (5) K6-89

Public Reading Room (5) Al-65

Publishing Coordination $\mathrm{K} 1-11$

Records Center

$\mathrm{K} 3-70$

Technical Library (2)

P8-55 